PROCEEDINGS OF THE

AMERICAN MATHEMATICAL SOCIETY

Volume 126, Number 8, August 1998, Pages 2417-2425

S $0002-9939(98) 04327-5$

\title{
NORMS OF EMBEDDINGS OF LOGARITHMIC BESSEL POTENTIAL SPACES
}

\author{
DAVID E. EDMUNDS, PETR GURKA, AND BOHUMÍR OPIC \\ (Communicated by Palle E. T. Jorgensen)
}

\begin{abstract}
Let $\Omega$ be a subset of $\mathbb{R}^{n}$ with finite volume, let $\nu>0$ and let $\Phi$ be a Young function with $\Phi(t)=\exp \left(\exp t^{\nu}\right)$ for large $t$. We show that the norm on the Orlicz space $L_{\Phi}(\Omega)$ is equivalent to

$$
\sup _{1<q<\infty}(e+\log q)^{-1 / \nu}\|f\|_{L^{q}(\Omega)}
$$

We also obtain estimates of the norms of the embeddings of certain logarithmic Bessel potential spaces in $L^{q}(\Omega)$ which are sharp in their dependences on $q$ provided that $q$ is large enough.
\end{abstract}

\section{INTRODUCTION}

In a series of recent papers ([EGO II], [EGO III], [EGO IV], [EOP]) an intensive study has been made of the logarithmic Bessel potential spaces

$$
H^{\sigma} L_{p, q ; \alpha, \beta}\left(\mathbb{R}^{n}\right):=\left\{u=g_{\sigma} * f ; f \in L_{p, q ; \alpha, \beta}\left(\mathbb{R}^{n}\right)\right\} .
$$

Here $\sigma>0, p \in(1, \infty), q \in[1, \infty]$, and $\alpha, \beta \in \mathbb{R} ; g_{\sigma}$ is the usual Bessel kernel and $L_{p, q ; \alpha, \beta}\left(\mathbb{R}^{n}\right)$ is the generalized Lorentz-Zygmund (GLZ) space of all functions $f$ on $\mathbb{R}^{n}$ such that

$$
\left\|t^{1 / p-1 / q}(e+|\log t|)^{\alpha} \log ^{\beta}(e+|\log t|) f^{*}(t)\right\|_{L^{q}(0, \infty)}<\infty,
$$

$f^{*}$ being the non-increasing rearrangement of $f$. (In Section 2 we shall see that GLZ spaces include many familiar objects including Lebesgue, Lorentz, LorentzZygmund, and Zygmund spaces.) A good deal of this work was motivated by the need for embedding theorems more delicate than the classical ones and involving scales of spaces which could be more finely tuned than the Lebesgue scale. We refer to $[\mathrm{ET}]$ for applications of related ideas to the distribution of eigenvalues of degenerate elliptic operators.

One of the theorems obtained in [EGO II] (see also [EGO IV]) concerned the embedding of certain GLZ spaces in Orlicz spaces of double exponential type. To explain this, suppose that $\Omega$ is a subset of $\mathbb{R}^{n}$ with finite volume, let $\nu>0$

Received by the editors January 23, 1997.

1991 Mathematics Subject Classification. Primary 46E35, 46E30.

Key words and phrases. Generalized Lorentz-Zygmund spaces, logarithmic Bessel potential spaces, Orlicz spaces of double and single exponential types, equivalent norms, embeddings.

This research was partially supported by grant no. 201/94/1066 of the Grant Agency of the Czech Republic and by NATO Collaborative Research Grant no. CRG 930358; the research of the second author was also partially supported by EPSRC grant no. GR/L02937.

(C) 1998 American Mathematical Society 
and denote by $E E_{\nu}(\Omega)$ the Orlicz space $L_{\Phi}(\Omega)$ with Young function $\Phi$ given by $\Phi(t)=\exp \left(\exp t^{\nu}\right)$ for large $t$. It turns out that when $0<\sigma<n$ and $\beta<1 / p^{\prime}$, then $H^{\sigma} L_{n / \sigma, p ; 1 / p^{\prime}, \beta}\left(\mathbb{R}^{n}\right)$ is continuously embedded in $E E_{\nu}(\Omega)$, where $1 / \nu=1 / p^{\prime}-\beta$. Another specialization of the general results of [EGO I, EGO II, EGO IV] gives analogous results concerning embeddings into Orlicz space of single exponential type, linking up with the celebrated embedding theorem of Trudinger $[\mathrm{T}]$ and Strichartz [S] and the more recent one due to Fusco, Lions and Sbordone [FLS].

The main results in the present paper are:

(a) We show that when $\nu>0$ and $\Omega \subset \mathbb{R}^{n}$ has finite volume, then a norm on $E E_{\nu}(\Omega)$ equivalent to the usual Orlicz norm is

$$
\sup _{1<q<\infty}(e+\log q)^{-1 / \nu}\|f\|_{L^{q}(\Omega)} .
$$

This reduces the derivation of estimates in $E E_{\nu}(\Omega)$ to that of $L^{q}(\Omega)$-estimates. The result should be compared with that in Orlicz spaces of single exponential type (see, for example, $[\operatorname{Tr}])$, in which $(e+\log q)$ is replaced by $q$.

(b) We obtain estimates for the norms of the embeddings of

$$
H^{\sigma} L_{n / \sigma, p ; 1 / p^{\prime}, \beta}\left(\mathbb{R}^{n}\right) \text { and } H^{\sigma} L_{n / \sigma, p ; \beta}\left(\mathbb{R}^{n}\right) \text { in } L^{q}(\Omega)
$$

(when $\Omega$ has finite volume and $\beta<1 / p^{\prime}$ ) which are sharp in their dependence on $q$ for large values of $q$.

The analogues of (a) and (b) in the context of single exponential spaces have proved to be very useful in the estimation of the asymptotic behaviour of eigenvalues of degenerate elliptic operators by means of entropy number estimates (see [ET]). We anticipate that our results will lead to similar developments in which spaces of double exponential type play a prominent role.

\section{NOTATION AND PRELIMINARIES}

Let $\Omega$ be a measurable subset of $\mathbb{R}^{n}$ (with respect to $n$-dimensional Lebesgue measure); by $|\Omega|_{n}$ we mean its $n$-volume; $\chi_{\Omega}$ will represent the characteristic function of $\Omega$.

For a measurable (real or complex) function $f$ on $\Omega$, its distribution function $\mu_{f}$ is given by

$$
\mu_{f}(\lambda)=\mu_{f, \Omega}(\lambda):=|\{x \in \Omega ;|f(x)|>\lambda\}|_{n}, \quad \lambda>0,
$$

and the non-increasing rearrangement $f^{*}$ of $f$ is defined by

$$
f^{*}(t)=f_{\Omega}^{*}(t):=\inf \left\{\lambda>0 ; \mu_{f, \Omega}(\lambda) \leq t\right\}, \quad t>0 .
$$

If $p, q \in(0, \infty]$ and $\alpha, \beta \in \mathbb{R}$, the generalized Lorentz-Zygmund space $L_{p, q ; \alpha, \beta}(\Omega)$ consists of all functions $f$ on $\Omega$ such that

$$
\begin{aligned}
\|f\|_{p, q ; \alpha, \beta} & =\|f\|_{p, q ; \alpha, \beta ; \Omega} \\
& =\left\|t^{1 / p-1 / q}(e+|\log t|)^{\alpha} \log ^{\beta}(e+|\log t|) f_{\Omega}^{*}(t)\right\|_{q ;(0, \infty)}<\infty,
\end{aligned}
$$

where $\|\cdot\|_{q ;(0, \infty)}$ stands for the $L^{q}$-(quasi-) norm on $(0, \infty)$.

When $\alpha=\beta=0$, the space $L_{p, q ; \alpha, \beta}(\Omega)$ coincides with the classical Lorentz space $L^{p, q}(\Omega)$, which is just $L^{p}(\Omega)$ when $q=p ; L_{p, q ; \alpha}(\Omega):=L_{p, q ; \alpha, 0}(\Omega)$ is the LorentzZygmund space $L^{p, q}(\log L)^{\alpha}(\Omega)$ introduced in [BR] and which, when $p=q$, is the Zygmund space $L^{p}(\log L)^{\alpha}(\Omega)$. If $p=q<\infty$ and $|\Omega|_{n}<\infty$, then $L_{p, q ; \alpha, \beta}(\Omega)$ equals

$$
L^{p}(\log L)^{\alpha}(\log \log L)^{\beta}(\Omega)=\left\{f ; \int_{\Omega}|f|^{p} \log ^{\alpha p}(e+|f|) \cdot[\log \log (2 e+|f|)]^{\beta p}<\infty\right\} .
$$


These spaces were studied in [EGO II] and in [EOP], where the properties mentioned above can be found together with some other information.

The Bessel kernel $g_{\sigma}(\sigma>0)$ is defined to be that function on $\mathbb{R}^{n}$ whose Fourier transform is

$$
\hat{g}_{\sigma}(x)=(2 \pi)^{-n / 2}\left(1+|x|^{2}\right)^{-\sigma / 2},
$$

where by the Fourier transform $\hat{f}$ of a function $f$ we mean

$$
\hat{f}(x)=(2 \pi)^{-n / 2} \int_{\mathbb{R}^{n}} e^{-i x \cdot y} f(y) d y .
$$

It is known that $g_{\sigma}$ is a positive, integrable function which is analytic except at the origin (cf. $[\mathrm{AS}]$ or $[\mathrm{Z}]$ ). We can now define the Bessel potential space with which we shall be concerned in this paper.

Let $\sigma>0, p \in(1, \infty), q \in[1, \infty]$, and $\alpha, \beta \in \mathbb{R}$. The logarithmic Bessel potential space $H^{\sigma} L_{p, q ; \alpha, \beta}\left(\mathbb{R}^{n}\right)$ is defined by

$$
H^{\sigma} L_{p, q ; \alpha, \beta}\left(\mathbb{R}^{n}\right):=\left\{u=g_{\sigma} * f ; f \in L_{p, q ; \alpha, \beta}\left(\mathbb{R}^{n}\right)\right\},
$$

and is equipped with the (quasi-) norm

$$
\|u\|_{\sigma ; p, q ; \alpha, \beta}:=\|f\|_{p, q ; \alpha, \beta} \text {. }
$$

Note that $H^{\sigma} L_{p, p ; 0,0}\left(\mathbb{R}^{n}\right)$ is simply the (fractional) Sobolev space of order $\sigma$.

When $k \in \mathbb{N}, p, q \in(1, \infty)$ and $\alpha, \beta \in \mathbb{R}$, then, by [EGO IV, Theorem 4.2], the space $H^{k} L_{p, q ; \alpha, \beta}\left(\mathbb{R}^{n}\right)$ is equal to the logarithmic Sobolev space

$$
W^{k} L_{p, q ; \alpha, \beta}\left(\mathbb{R}^{n}\right):=\left\{u ; D^{\alpha} u \in L_{p, q ; \alpha, \beta}\left(\mathbb{R}^{n}\right) \text { if }|\alpha| \leq k\right\},
$$

equipped with the (quasi-) norm

$$
\sum_{|\alpha| \leq k}\left\|D^{\alpha} u\right\|_{p, q ; \alpha, \beta}
$$

and the corresponding (quasi-) norms are equivalent.

By a Young function $\Phi$ we shall mean a continuous, non-negative, strictly increasing, convex function on $[0, \infty)$ such that

$$
\lim _{t \rightarrow 0^{+}} \frac{\Phi(t)}{t}=\lim _{t \rightarrow \infty} \frac{t}{\Phi(t)}=0 .
$$

Given a Young function $\Phi$ and any measurable subset $\Omega$ of $\mathbb{R}^{n}, L_{\Phi}(\Omega)$ will denote the corresponding Orlicz space, equipped with the Luxemburg norm $\|\cdot\|_{\Phi ; \Omega}$; for details of such spaces we refer to $[\mathrm{A}],[\mathrm{KJF}],[\mathrm{BS}]$.

Lemma 2.1. Let $\Omega \subset \mathbb{R}^{n}$ be a domain with $|\Omega|_{n}<\infty$ and let $\nu>0$. Then:

(i) The space $L^{\infty}(\log L)^{-1 / \nu}(\Omega)=L_{\infty, \infty ;-1 / \nu}(\Omega)$ coincides with the Orlicz space $L_{\Phi}(\Omega)$, where $\Phi(t)=\exp t^{\nu}$ for all $t \geq t_{0}$ with some $t_{0} \in(0, \infty)$, and the corresponding (quasi-) norms are equivalent.

(ii) The space $L^{\infty}(\log \log L)^{-1 / \nu}(\Omega)=L_{\infty, \infty ; 0,-1 / \nu}(\Omega)$ coincides with the Orlicz space $L_{\Phi}(\Omega)$, where $\Phi(t)=\exp \exp t^{\nu}$ for all $t \geq t_{0}$ with some $t_{0} \in(0, \infty)$, and the corresponding (quasi-) norms are equivalent.

Proof. The proof of (i) can be found in [BR, Theorem D]; statement (ii) can be proved similarly (cf. [EGO I, Lemma 3.10]). The assertion about equivalent norms easily follows from [BS, Chapter 1, Thm. 1.8] and from the fact that all the spaces are (equivalent to) Banach function spaces. 
The lemma leads us to the following notation.

Notation 2.2. Let $\Omega \subset \mathbb{R}^{n},|\Omega|_{n}<\infty$ and $\nu>0$. Then we put

$$
\begin{gathered}
E_{\nu}(\Omega):=L^{\infty}(\log L)^{-1 / \nu}(\Omega)=L_{\infty, \infty ;-1 / \nu}(\Omega), \\
\|\cdot\|_{E_{\nu}(\Omega)}:=\|\cdot\|_{\infty, \infty ;-1 / \nu ; \Omega} ; \\
E E_{\nu}(\Omega):=L^{\infty}(\log \log L)^{-1 / \nu}(\Omega)=L_{\infty, \infty ; 0,-1 / \nu}(\Omega), \\
\|\cdot\|_{E E_{\nu}(\Omega)}:=\|\cdot\|_{\infty, \infty ; 0,-1 / \nu ; \Omega} .
\end{gathered}
$$

For non-negative expressions (i.e. functions or functionals) $F_{1}, F_{2}$ we use the symbols $F_{1} \lesssim F_{2}$ and $F_{1} \gtrsim F_{2}$, respectively, provided that $F_{1} \leq C F_{2}$ and $C F_{1} \geq F_{2}$, with some constant $C \in(0, \infty)$ independent of the variables in the expressions $F_{1}$, $F_{2}$. If $F_{1} \lesssim F_{2}$ and $F_{1} \gtrsim F_{2}$, we write $F_{1} \approx F_{2}$.

\section{EquiVAlent NORMS in EXPONENTIAL SPACES}

Theorem 3.1. Let $\nu>0$, and let $\Omega \subset \mathbb{R}^{n}$ be a domain with $|\Omega|_{n}<\infty$. Then

$$
\|f\|_{E_{\nu}(\Omega)} \approx \sup _{q \in(1, \infty)} q^{-1 / \nu}\|f\|_{q ; \Omega} \quad \text { for all } f \in E_{\nu}(\Omega)
$$

and

$$
\|f\|_{E E_{\nu}(\Omega)} \approx \sup _{q \in(1, \infty)}(e+\log q)^{-1 / \nu}\|f\|_{q ; \Omega} \quad \text { for all } f \in E E_{\nu}(\Omega) .
$$

Proof. We prove only (3.2); for the proof of (3.1) see e.g. [Tr]. The proof will be given in two steps.

STEP 1. We show that

$$
\|f\|_{E E_{\nu}(\Omega)} \approx \sup _{\sigma \in(0,1)}(e-\log \sigma)^{-1 / \nu}\|f\|_{\frac{1}{\sigma}, \infty ; \Omega} .
$$

(Recall that $\|\cdot\|_{\frac{1}{\sigma}, \infty ; \Omega}$ denotes the (quasi-) norm in the Lorentz (Marcinkiewicz) $L^{\frac{1}{\sigma}, \infty}(\Omega)$-space, i.e. $\|f\|_{1 / \sigma, \infty ; \Omega}=\sup _{s \in\left(0,|\Omega|_{n}\right)} s^{\sigma} f_{\Omega}^{*}(s)$.)

First we show the relation " $>$ ". Suppose that $f \in E E_{\nu}(\Omega)$, i.e.

$$
\sup _{s \in\left(0,|\Omega|_{n}\right)} \log ^{-1 / \nu}(e+|\log s|) f_{\Omega}^{*}(s)<\infty .
$$

It is plain that, for $\sigma \in(0,1)$,

$$
(e-\log \sigma)^{-1 / \nu} \approx\left(\frac{2}{\nu}-\log \sigma\right)^{-1 / \nu},
$$

and thus

$$
\begin{aligned}
(e-\log \sigma)^{-1 / \nu}\|f\|_{\frac{1}{\sigma}, \infty ; \Omega} & \approx\left(\frac{2}{\nu}-\log \sigma\right)^{-1 / \nu} \sup _{s \in\left(0,|\Omega|_{n}\right)} s^{\sigma} f_{\Omega}^{*}(s) \\
& =\sup _{s \in\left(0,|\Omega|_{n}\right)}\left(\frac{2}{\nu}-\log \sigma\right)^{-1 / \nu} s^{\sigma} f_{\Omega}^{*}(s) .
\end{aligned}
$$

We prove the estimate

$$
\left(\frac{2}{\nu}-\log \sigma\right)^{-1 / \nu} s^{\sigma} \leq C \log ^{-1 / \nu}(e+|\log s|)
$$

with a constant $C>0$ independent of $\sigma \in(0,1)$ and $s \in\left(0,|\Omega|_{n}\right)$. 
If $|\Omega|_{n}>1$, then, for any $s \in\left[1,|\Omega|_{n}\right)$ and $\sigma \in(0,1)$,

$$
\left(\frac{2}{\nu}-\log \sigma\right)^{-1 / \nu} s^{\sigma} \leq\left(\frac{2}{\nu}\right)^{-1 / \nu}|\Omega|_{n} \leq C \log ^{-1 / \nu}(e+|\log s|)
$$

with $C>0$ independent of $\sigma$ and $s$. So we can assume that $|\Omega|_{n} \leq 1$.

Let $s \in(0,1)$ be fixed. For $\tau \in(0,-\log s)$ define

$$
F_{s}(\tau)=\left(\frac{2}{\nu}+\log (-\log s)-\log \tau\right)^{-1 / \nu} e^{-\tau}
$$

Then

$$
F_{s}(\tau) \leq C \log ^{-1 / \nu}(e-\log s)
$$

with $C$ independent of $\tau$ and $s$. Indeed, if $0<\tau \leq \frac{1}{2}$, then (3.7) holds since $(-\log \tau)>0,-\frac{1}{\nu}<0$ and $e^{-\tau}<1$. If $\frac{1}{2}<\tau<-\log s$, then the first derivative

$$
\frac{d F_{s}}{d \tau}(\tau)=\frac{e^{-\tau}}{\tau}\left(\frac{2}{\nu}+\log (-\log s)-\log \tau\right)^{-\frac{1}{\nu}-1}\left[\frac{1}{\nu}-\tau\left(\frac{2}{\nu}+\log (-\log s)-\log \tau\right)\right]
$$

is negative, since

$$
\frac{1}{\nu}-\tau\left(\frac{2}{\nu}+\log (-\log s)-\log \tau\right)<\frac{1}{\nu}-\tau \cdot \frac{2}{\nu}<0 .
$$

Hence, the function $F_{s}$ is decreasing on the interval $\left[\frac{1}{2},-\log s\right)$, which implies that for any $\tau \in\left(\frac{1}{2},-\log s\right)$,

$$
F_{s}(\tau) \leq F_{s}\left(\frac{1}{2}\right)=\left(\frac{2}{\nu}+\log (-\log s)+\log 2\right)^{-1 / \nu} e^{-\frac{1}{2}} \leq C \log ^{-1 / \nu}(e-\log s)
$$

with $C$ independent of $\tau$ and $s$, and (3.7) is proved.

Now, putting $\tau=\sigma(-\log s)$ with $\sigma \in(0,1)$, we obtain from $(3.7)$ that

$$
\begin{aligned}
& C \log ^{-1 / \nu}(e-\log s) \geq F_{s}(\sigma(-\log s)) \\
& \quad=\left(\frac{2}{\nu}+\log (-\log s)-\log (\sigma(-\log s))\right)^{-1 / \nu} e^{\sigma \log s}=\left(\frac{2}{\nu}-\log \sigma\right)^{-1 / \nu} s^{\sigma} .
\end{aligned}
$$

Together with (3.6), this verifies (3.5). The desired inequality follows from (3.4) and (3.5).

We next prove the relation "¿" in $(3.3)$. Suppose that

$$
\sup _{\sigma \in(0,1)}(e-\log \sigma)^{-1 / \nu}\|f\|_{\frac{1}{\sigma}, \infty ; \Omega}=: A<\infty .
$$

Fix $s \in\left(0,|\Omega|_{n}\right)$ and set $\sigma=1 /(e+|\log s|)$. Then $\sigma \in(0,1)$, and we have, for any $s \in\left(0,|\Omega|_{n}\right)$,

$$
\begin{aligned}
A & \geq(e-\log \sigma)^{-1 / \nu}\|f\|_{\frac{1}{\sigma}, \infty ; \Omega} \geq(e-\log \sigma)^{-1 / \nu} s^{\sigma} f_{\Omega}^{*}(s) \\
& =(e+\log (e+|\log s|))^{-1 / \nu} \exp \left(\frac{\log s}{e+|\log s|}\right) f_{\Omega}^{*}(s) \\
& \geq C \log ^{-1 / \nu}(e+|\log s|) f_{\Omega}^{*}(s)
\end{aligned}
$$

with a constant $C$ independent of $s$. Passing to the supremum over all $s \in\left(0,|\Omega|_{n}\right)$, we obtain the desired estimate, and (3.3) is proved.

STEP 2. First we prove that

$$
\sup _{\sigma \in(0,1)}(e-\log \sigma)^{-1 / \nu}\|f\|_{\frac{1}{\sigma}, \infty ; \Omega} \approx \sup _{\sigma \in(0,1)}(e-\log \sigma)^{-1 / \nu}\|f\|_{\frac{1}{\sigma} ; \Omega}
$$

(recall that $\|\cdot\|_{p ; \Omega}$ stands for the norm in the space $L^{p}(\Omega)$ ). 
Assuming that

$$
\sup _{s \in(0,1)}(e-\log \sigma)^{-1 / \nu}\|f\|_{\frac{1}{\sigma} ; \Omega}=: B<\infty
$$

and using the well-known inequality

$$
\|f\|_{\frac{1}{\sigma}, \infty ; \Omega} \leq\|f\|_{\frac{1}{\sigma} ; \Omega}
$$

we immediately obtain

$$
\sup _{\sigma \in(0,1)}(e-\log \sigma)^{-1 / \nu}\|f\|_{\frac{1}{\sigma}, \infty ; \Omega} \leq B
$$

We prove the converse inequality. If $0<\sigma_{1}<\sigma$, then

$$
\begin{aligned}
\|f\|_{\frac{1}{\sigma} ; \Omega} & =\left(\int_{0}^{|\Omega|_{n}}\left(f_{\Omega}^{*}(t)\right)^{\frac{1}{\sigma}} d t\right)^{\sigma}=\left(\int_{0}^{|\Omega|_{n}} t^{1-\frac{\sigma_{1}}{\sigma}}\left(t^{\sigma_{1}} f_{\Omega}^{*}(t)\right)^{\frac{1}{\sigma}} \frac{d t}{t}\right)^{\sigma} \\
& \leq\left(1-\frac{\sigma_{1}}{\sigma}\right)^{-\sigma}|\Omega|_{n}^{\sigma-\sigma_{1}}\|f\|_{\frac{1}{\sigma_{1}}, \infty ; \Omega} .
\end{aligned}
$$

The estimate

$$
(e-\log \sigma)^{-1 / \nu} \approx(e+\log 2-\log \sigma)^{-1 / \nu}=\left(e-\log \frac{\sigma}{2}\right)^{-1 / \nu}, \quad \sigma \in(0,1),
$$

and (3.9) with $\sigma_{1}=\sigma / 2$ imply

$$
(e-\log \sigma)^{-1 / \nu}\|f\|_{\frac{1}{\sigma} ; \Omega} \leq C\left(e-\log \sigma_{1}\right)^{-1 / \nu}\|f\|_{\frac{1}{\sigma_{1}}, \infty ; \Omega}, \quad \sigma \in(0,1),
$$

where $C$ is independent of $f$. Consequently,

$$
\begin{aligned}
\sup _{\sigma \in(0,1)}(e-\log \sigma)^{-1 / \nu}\|f\|_{\frac{1}{\sigma} ; \Omega} & \lesssim \sup _{\sigma_{1} \in\left(0, \frac{1}{2}\right)}\left(e-\log \sigma_{1}\right)^{-1 / \nu}\|f\|_{\frac{1}{\sigma_{1}}, \infty ; \Omega} \\
& \lesssim \sup _{\sigma \in(0,1)}(e-\log \sigma)^{-1 / \nu}\|f\|_{\frac{1}{\sigma}, \infty ; \Omega}
\end{aligned}
$$

and (3.8) is proved.

Now, (3.2) easily follows from (3.3) and (3.8) on putting $q=1 / \sigma$.

Corollary 3.2. Let $|\Omega|_{n}<\infty$ and $\nu>0$.

(i) If $j_{0} \in \mathbb{N}$ and $q_{0} \geq 1$, then, for all $f \in E_{\nu}(\Omega)$,

$$
\|f\|_{E_{\nu}(\Omega)} \approx \sup _{j \in \mathbb{N}, j \geq j_{0}} j^{-1 / \nu}\|f\|_{j ; \Omega} \approx \sup _{q \geq q_{0}} q^{-1 / \nu}\|f\|_{q ; \Omega} .
$$

(ii) If $j_{0} \in \mathbb{N}, j_{0} \geq 2$ and $q_{0}>1$, then, for all $f \in E E_{\nu}(\Omega)$,

$$
\|f\|_{E E_{\nu}(\Omega)} \approx \sup _{j \in \mathbb{N}, j \geq j_{0}}(\log j)^{-1 / \nu}\|f\|_{j ; \Omega} \approx \sup _{q \geq q_{0}}(\log q)^{-1 / \nu}\|f\|_{q ; \Omega} .
$$

Proof. We prove only (ii); the proof of (i) is analogous. 
Let $q_{0}>1$ and $j_{0} \geq 2$ be fixed. For $f \in E E_{\nu}(\Omega)$ we denote

$$
\begin{aligned}
S_{1}(f) & =\sup _{q \in(1, \infty)}(e+\log q)^{-1 / \nu}\|f\|_{q ; \Omega}, \\
S_{2}(f) & =\sup _{q \in(1, \infty)}(e+\log q)^{-1 / \nu}|\Omega|_{n}^{-1 / q}\|f\|_{q ; \Omega}, \\
S_{3}(f) & =\sup _{q \geq q_{0}}(\log q)^{-1 / \nu}\|f\|_{q ; \Omega}, \\
\mathfrak{S}_{1}(f) & =\sup _{j \in \mathbb{N}, j \geq j_{0}}(\log j)^{-1 / \nu}\|f\|_{j ; \Omega}, \\
\mathfrak{S}_{2}(f) & =\sup _{j \in \mathbb{N}, j \geq j_{0}}(\log j)^{-1 / \nu}|\Omega|_{n}^{-1 / j}\|f\|_{j ; \Omega}, \\
\mathfrak{S}_{3}(f) & =\sup _{j \in \mathbb{N}, j \geq\left[q_{0}\right]+1}(\log j)^{-1 / \nu}\|f\|_{j ; \Omega} .
\end{aligned}
$$

If $q \in(1, \infty)$, we put $j=\max \left\{j_{0},[q]+1\right\}$ (where $[q]$ denotes the integer part of $q$ ) and choose $k \in \mathbb{N}, k \geq 2$, satisfying $2^{k} \geq j_{0}$. Then

$$
j \leq j_{0}([q]+1) \leq j_{0}(q+1) \leq(4 q)^{k} \leq\left(e^{e} q\right)^{k}
$$

and hence

$$
(\log j)^{-1 / \nu} \geq k^{-1 / \nu}(e+\log q)^{-1 / \nu} .
$$

Moreover, by the Hölder inequality, for any $f \in E E_{\nu}(\Omega)$,

$$
|\Omega|_{n}^{-1 / q}\|f\|_{q ; \Omega} \leq|\Omega|_{n}^{-1 / j}\|f\|_{j ; \Omega},
$$

which together with (3.11) implies that

$$
S_{2}(f) \leq k^{1 / \nu} \mathfrak{S}_{2}(f) .
$$

Further, it is obvious that, for any $f \in E E_{\nu}(\Omega)$,

$$
S_{1}(f) \approx S_{2}(f), \quad \mathfrak{S}_{1}(f) \approx \mathfrak{S}_{2}(f),
$$

and since $(e+\log j) \approx \log j, j \geq j_{0}$, we have

$$
\mathfrak{S}_{1}(f) \lesssim S_{1}(f)
$$

Together with (3.12) these estimates imply

$$
\mathfrak{S}_{1}(f) \lesssim S_{1}(f) \approx S_{2}(f) \lesssim \mathfrak{S}_{2}(f) \approx \mathfrak{S}_{1}(f) \text { for all } f \in E E_{\nu}(\Omega) .
$$

The first relation in (3.10) follows from Theorem 3.1 and (3.13). Moreover, by Theorem 3.1, $\mathfrak{S}_{3}(f) \lesssim\|f\|_{E E_{\nu}(\Omega)}$. Since $\mathfrak{S}_{3}(f)=\mathfrak{S}_{1}(f)$ if $j_{0}:=\left[q_{0}\right]+1$, we have from the first relation in (3.10) that $\|f\|_{E E_{\nu}(\Omega)} \lesssim \mathfrak{S}_{3}(f)$, and the result follows.

\section{Norms OF EMBEDDINGS}

Given two (quasi-) Banach spaces $X$ and $Y$, we write $X \hookrightarrow Y$ if $X \subset Y$ and the natural embedding id : $X \rightarrow Y$ is continuous. The norm of the embedding is

$$
\|\mathrm{id}\|=\|\mathrm{id}\|_{X \rightarrow Y}=\sup _{\|f\|_{X} \leq 1}\|f\|_{Y}
$$

In this section we shall consider embeddings of Bessel potential spaces (modelled on generalized Lorentz-Zygmund spaces) into $L^{q}$-spaces. Our aim will be to establish the dependence of norms of these embeddings on $q$. 
Theorem 4.1. Let $0<\sigma<n, 1 \leq p \leq \infty$ and $\beta<1 / p^{\prime}$ (where $1 / p^{\prime}=1-1 / p$ ). Suppose that $\Omega \subset \mathbb{R}^{n}$ has non-empty interior and $|\Omega|_{n}<\infty$. Denote

$$
X_{1}=H^{\sigma} L_{\frac{n}{\sigma}, p ; \beta}\left(\mathbb{R}^{n}\right), \quad X_{2}=H^{\sigma} L_{\frac{n}{\sigma}, p ; \frac{1}{p^{\prime}}, \beta}\left(\mathbb{R}^{n}\right) .
$$

Then

$$
\begin{array}{rlrl}
\|\operatorname{id}\|_{X_{1} \rightarrow L^{q}(\Omega)} & \lesssim q^{1 / p^{\prime}-\beta}, & & 1<q<\infty, \\
\|\operatorname{id}\|_{X_{2} \rightarrow L^{q}(\Omega)} & \lesssim(e+\log q)^{1 / p^{\prime}-\beta}, & 1<q<\infty .
\end{array}
$$

Moreover, there is $q_{0} \in(1, \infty)$ such that

$$
\begin{array}{ll}
\|\mathrm{id}\|_{X_{1} \rightarrow L^{q}(\Omega)} & \approx q^{1 / p^{\prime}-\beta} \quad \text { for all } q>q_{0}, \\
\|\operatorname{id}\|_{X_{2} \rightarrow L^{q}(\Omega)} \approx(\log q)^{1 / p^{\prime}-\beta} & \text { for all } q>q_{0} .
\end{array}
$$

Proof. We prove only (4.2) and $\left(4.2^{*}\right)$; the proof of $(4.1)$ and $\left(4.1^{*}\right)$ is similar and is left to the reader.

Let the assumptions of Theorem 4.1 be satisfied. Then, by [EGO II, Theorem $5.2]$,

$$
H^{\sigma} L_{\frac{n}{\sigma}, p ; \frac{1}{p^{\prime}}, \beta}\left(\mathbb{R}^{n}\right) \hookrightarrow E E_{\alpha}(\Omega),
$$

where $1 / \alpha=1 / p^{\prime}-\beta>0$, i.e.,

$$
\|f\|_{E E_{\alpha}(\Omega)} \leq C<\infty
$$

for any $f \in X_{2}$ such that $\|f\|_{X_{2}} \leq 1$, with a constant $C$ independent of $f$. By (4.3) and $(3.2)$, for any $q \in(1, \infty)$,

$$
(e+\log q)^{-1 / \alpha}\|f\|_{q ; \Omega} \leq C,
$$

i.e.

$$
\|f\|_{q ; \Omega} \leq C(e+\log q)^{1 / p^{\prime}-\beta} .
$$

Passing to the supremum over all $f \in X_{2}$ with $\|f\|_{X_{2}} \leq 1$, we get (4.2).

Without loss of generality we can assume that the ball $B_{1}=\left\{x \in \mathbb{R}^{n} ;|x|<1\right\} \subset$ $\Omega$. By [EGO III, Lemma 4.3 and Remark 4.5] there exist $r_{1} \in(0,1)$ and a function $f_{r}, r \in\left(0, r_{1}\right)$, satisfying

$$
\begin{gathered}
\operatorname{supp} f_{r} \subset B_{1}, \\
\left|\left(g_{\sigma} * f_{r}\right)(x)\right| \geq C \log ^{1 / p^{\prime}-\beta}(e-\log r) \quad \text { for all }|x|<r
\end{gathered}
$$

(with $C \in(0, \infty)$ independent of $r$ ), and

$$
\left\|f_{r}\right\|_{\frac{n}{\sigma}, p ; \frac{1}{p^{\prime}}, \beta} \leq 1 .
$$

Choose $q_{0} \in(1, \infty)$ such that $\exp \left(e-q_{0}\right) \leq r_{1}$. If $q \in\left(q_{0}, \infty\right)$, set $r=\exp (e-q)$. Obviously, $r \in\left(0, r_{1}\right)$, and by (4.5) and (4.4) the function $F_{q}:=g_{\sigma} * f_{r}$ satisfies,

$$
\begin{gathered}
F_{q} \in H^{\sigma} L_{\frac{n}{\sigma}, p ; \frac{1}{p^{\prime}}, \beta}\left(\mathbb{R}^{n}\right), \quad\left\|F_{q}\right\|_{\sigma ; \frac{n}{\sigma}, p ; \frac{1}{p^{\prime}}, \beta} \leq 1, \\
\left\|F_{q}\right\|_{q ; \Omega} \geq\left(\int_{\{|x|<r\}}\left|F_{q}(x)\right|^{q} d x\right)^{1 / q} \gtrsim(\log q)^{1 / p^{\prime}-\beta},
\end{gathered}
$$

and $\left(4.2^{*}\right)$ follows. 
Remark 4.1. If we take $\sigma=1, p=n$ and $\beta=0$, we obtain from Theorem 4.1 that for all large $q$,

$$
\begin{gathered}
\|\operatorname{id}\|_{W^{1, n}\left(\mathbb{R}^{n}\right) \rightarrow L^{q}(\Omega)} \approx q^{1 / n^{\prime}}, \\
\|\operatorname{id}\|_{W^{1} L^{n}(\log L)^{1 / n^{\prime}}\left(\mathbb{R}^{n}\right) \rightarrow L^{q}(\Omega)} \approx(\log q)^{1 / n^{\prime}} .
\end{gathered}
$$

\section{REFERENCES}

[A] R. A. Adams, Sobolev spaces, Academic Press, New York, 1975. MR 56:9247

[AS] N. Aronszajn and K. T. Smith, Theory of Bessel potentials, Part I, Ann. Inst. Fourier 11 (1961), 385-475. MR 26:1485

[BR] C. Bennett and K. Rudnick, On Lorentz-Zygmund spaces, Dissertationes Math. 175 (1980), 1-72. MR 81i:42020

[BS] C. Bennett and R. Sharpley, Interpolation of operators, Pure Appl. Math. 129, Academic Press, New York, 1988. MR 89e:46001

[EGO I] D. E. Edmunds, P. Gurka and B. Opic, Double exponential integrability of convolution operators in generalized Lorentz-Zygmund spaces, Indiana Univ. Math. J. 44 (1995), 19-43. MR 96f: 47048

[EGO II] D. E. Edmunds, P. Gurka and B. Opic, Double exponential integrability, Bessel potentials and embedding theorems, Studia Math. 115 (1995), 151-181. MR 96i:46036

[EGO III] D. E. Edmunds, P. Gurka and B. Opic, Sharpness of embeddings in logarithmic Besselpotential spaces, Proc. Roy. Soc. Edinburgh 126A (1996), 995-1009. CMP 97:03

[EGO IV] D. E. Edmunds, P. Gurka and B. Opic, On embeddings of logarithmic Bessel potential spaces, J. Functional Anal. 146 (1997), 116-150. CMP 97:12

[EOP] W. D. Evans, B. Opic and L. Pick, Interpolation of operators on scales of generalized Lorentz-Zygmund spaces, Math. Nachr. 182 (1996), 127-181. CMP 97:04

[ET] D. E. Edmunds and H. Triebel, Function spaces, entropy numbers and differential operators, Cambridge University Press, Cambridge, 1996, pp. 252. MR 97h:46045

[FLS] N. Fusco, P. L. Lions and C. Sbordone, Sobolev imbedding theorems in borderline cases, Proc. Amer. Math. Soc. 124 (2) (1996), 561-565. MR 96d:46035

[KJF] A. Kufner, O. John and S. Fučík, Function spaces, Academia, Prague, 1977. MR 58:2189

[S] R. S. Strichartz, A note on Trudinger's extension of Sobolev's inequalities, Indiana Univ. Math. J. 21 (1972), 841-842. MR 45:2466

[T] N. S. Trudinger, On imbeddings into Orlicz spaces and some applications, J. Math. Mech. 17 (1967), 473-484. MR 35:7121

[Tr] H. Triebel, Approximation numbers and entropy numbers of embeddings of fractional Besov-Sobolev spaces in Orlicz spaces, Proc. London Math. Soc. 66 (3) (1993), 589-618. MR 94g:46042

[Z] W. Ziemer, Weakly differentiable functions, Graduate Texts in Math. 120, Springer, Berlin, 1989. MR 91e:46046

Centre for Mathematical Analysis and its Applications, University of Sussex, FALmer, Brighton BN1 9QH, England

E-mail address: d.e.edmunds@sussex.ac.uk

Department of Mathematics, Czech University of Agriculture, 16521 Prague 6, Czech Republic

E-mail address: gurka@tf.czu.cz

Mathematical Institute, Academy of Sciences of the Czech Republic, Žitná 25, 11567

Prague 1, Czech Republic

E-mail address: opic@math.cas.cz 